Original Research Paper

\title{
Estimation for Unknown Parameters of the Burr Type-XII Distribution Based on an Adaptive Progressive Type-II Censoring Scheme
}

\author{
${ }^{1,2}$ Montaser M. Amein \\ ${ }^{1}$ Department of Mathematics, Faculty of Science, Al-Azhar University, Nasr City (11884), Cairo, Egypt \\ ${ }^{2}$ Department of Mathematics and Statistics, Faculty of Science, Taif University, Hawia (888), Taif, Kingdom of Saudi Arabia
}

\author{
Article history \\ Received: 16-02-2016 \\ Revised: 19-04-2016 \\ Accepted: 29-04-2016 \\ Email: montaser.m.amein@gmail.com
}

\begin{abstract}
In this study, the Maximum Likelihood Estimation (MLE) and Bayes estimation are exploited to make interval estimation based on adaptive progressive Type-II censoring for the Burr Type-XII distribution. Explicit form for the parameters of Bayes estimator doesn't exist, so, Markov Chain Monte Carlo (MCMC) method is used as approximation to find posterior mean under squared error loss function. Real data set are presented to illustrate the methods of inference.
\end{abstract}

Keywords: An Adaptive Type-II Progressive Censoring Scheme, Bayesian and Non-Bayesian Estimations, Gibbs and Metropolis Sampler, Burr TypeXII Distribution

\section{Introduction}

Censored sampling have been attracting great interest due to their wide applications. In life testing, the experimenter may not always obtain complete information on failure times for all experimental units. Reducing the total test time and the associated cost is one of the major reasons for censoring. A more general censoring scheme called progressive Type-II right censoring, in which $n$ independent items are put on life test with continuous, identically distributed failure times $X_{1}, X_{2}, \cdots, X_{n}$, with a censoring scheme $\left(R_{1}, R_{2}, \ldots, R_{m}\right)$ is prefixed (Fig. 1). Progressive censoring have studied by many authors. Among them are (Viveros and Balakrishnan, 1994; Aggarwala and Balakrishnan, 1996; Balakrishnan and Aggarwala, 2000; Wu, 2002; Balakrishnan et al., 2002; Balakrishnan, 2007; Ng and Chan, 2007). For extensive survey of progressive censored, see (Balakrishnan and Aggarwala, 2000).

\section{The Model Description}

Recently, $\mathrm{Ng}$ et al. (2010) have established an adaptive Type-II progressive censoring scheme. In which, a mixture of order statistics and Type-II progressive censoring schemes. For this censoring scheme, time $T$ and the number of observed failures $m$ is prefixed and $T>m$. Suppose $J$ is the number of failures observed before time $T$, i.e., $X_{J: m: n}<T<X_{J+1: m: n}, J=0,1$, $2, \cdots, m$ where $X_{0: m: n}=0, X_{m+1: m: n}=\infty$. After the experiment passed time $T$, set $R_{j+1}=\cdots=R_{m}=0$. Figure 2 gives the schematic representation of this situation.

Two cases for the value of $\mathrm{T}$ differ from the adaptive Type-II progressive censoring. First: $(m<T)$ : The conventional a usual progressive Type-II censoring scheme with $\left(R_{1}, R_{2}, \ldots, R_{m}\right)$. Second: Extreme case can occur when $T=0$, which results in the conventional Type-II censoring scheme $R_{1}=R_{2}=\ldots=R_{m-1}=0$ and $R_{m}$ $=n-m$. Many papers dealing with adaptive progressive Type-II censoring have been appeared. Among them, Soliman et al. $(2011$; 2012) have used a Bayes study using MCMC approach based on progressive censoring data. (For more details about MCMC; see, for example, Robert and Casella (2005) and Recently, Rezaei et al. (2010). Recently, Mahmoud et al. (2013) have estimated the unknown parameters of generalized Pareto under an adaptive Type-II progressive censoring.

If the failure times of the $n$ items originally on the test are from a Burr Type-XII (Burr-XII) population with cdf $F(x)$ and pdf $f(x)$ as follow:

$$
f(x)=\frac{c \beta x^{(c-1)}}{\left(1+x^{c}\right)^{\beta+1}}
$$

And:

$$
F(x)=1-\frac{1}{\left(1+x^{c}\right)^{\beta}}, \beta, c, x>0 .
$$




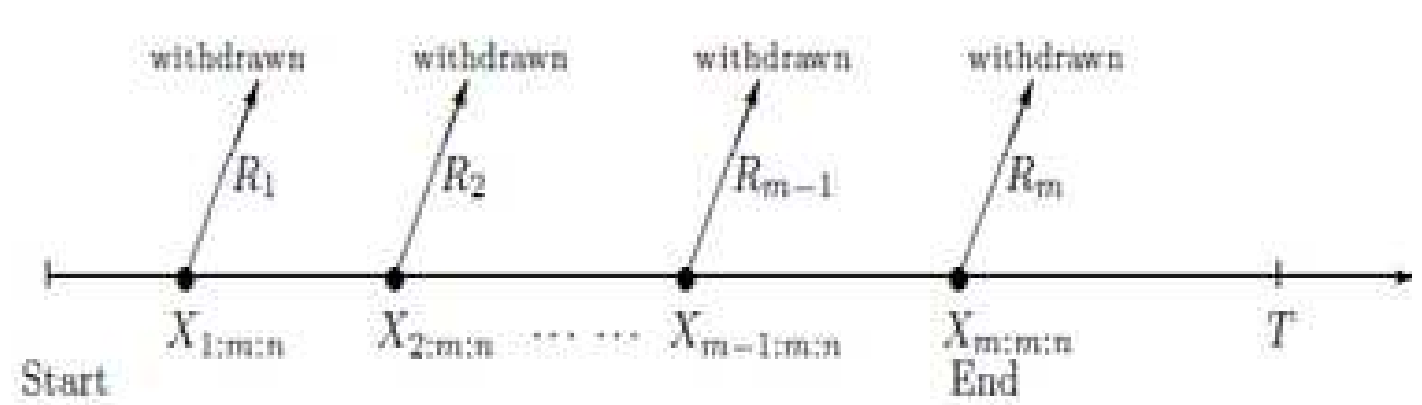

Fig. 1. Progressive Type-II censoring

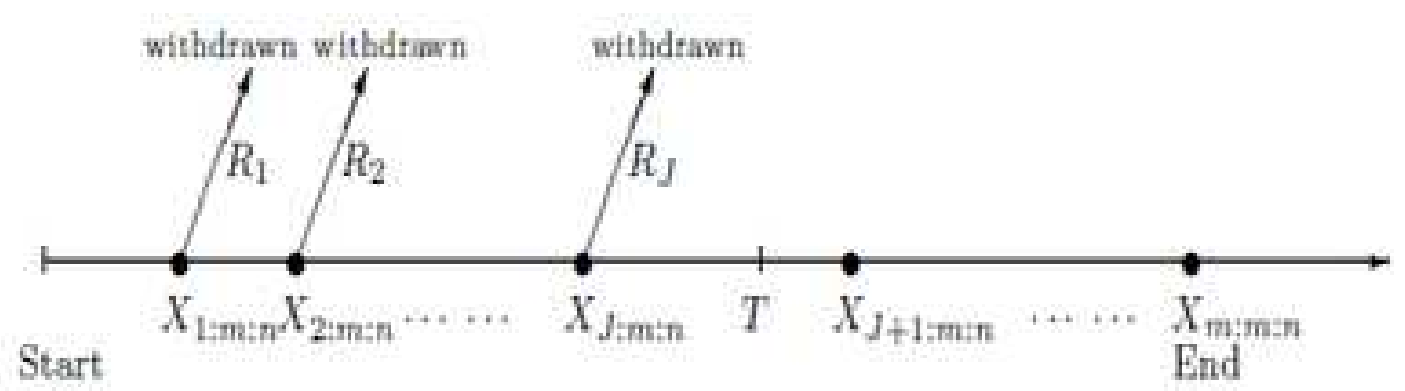

Fig. 2. Adaptive progressive Type-II censoring

In addition, the hazard rate function and reliability function are:

$$
H(x)=\frac{c \beta x^{(c-1)}}{\left(1+x^{c}\right)}
$$

And:

$$
S(x)=\frac{1}{\left(1+x^{c}\right)^{\beta}}
$$

Burr-XII distribution was first introduced in the literature by Burr (1942) and has special importance of using it in practical situations. Rodriguez (1977) showed that the Burr coverage area on a specific plane is occupied by various well known and useful distributions, including the normal, log-normal, gamma, logistic and extreme value type-I distributions. It has been applied in areas of reliability studies and failure time modeling. Other applications include simulation, approximation of distributions and development of non-normal control charts. Many authors have studied inferences on the Burr-XII distribution. Wingo (1993; Moore and Papadopoulos, 2000; Ali Mousa and Jaheen, 2002; Wu and Yu, 2005; Soliman, 2005; Xiuchun et al., 2007; Jaheen and Okasha, 2011; Wu et al., 2014).

\section{Maximum Likelihood Estimation (MLE)}

In this section, the confidence interval for the unknown parameters $c$ and $\beta$ are obtained using MLE technique. Let $x_{1: m: n}, x_{2: m: n}, \cdots, x_{m: m: n}$ be an adaptive type-II progressive censored order statistics from Burr-XII distribution with censoring scheme $\left\{R_{1}, R_{2}\right.$, $\left.R_{3}, \cdots, R_{m}\right\}$, then the joint probability density function can written as follows ( $\mathrm{Ng}$ et al., 2010):

$$
\begin{aligned}
& f_{1,2, \cdots, m: m: n}\left(x_{1}, x_{2}, \cdots, x_{m}\right)=d_{j}\left(\prod_{i=1}^{m} f\left(x_{i: m: n}\right)\right) \\
& \left(\prod_{i=1}^{j}\left(1-F\left(x_{i: m: n}\right)\right)\right)^{R_{i}}\left(1-F\left(x_{m: m: n}\right)\right)^{n-m-\sum_{i=1}^{j} R_{i}}, \\
& 0<x_{1: m: n}<x_{2: m: n}<\cdots<x_{m: m: n}<\infty
\end{aligned}
$$

Where:

$$
d_{j}=\prod_{i=1}^{m}\left(n-i+1-\sum_{k=1}^{\max (i-1, J)} R_{k}\right)
$$

The maximum likelihood for Burr-XII distribution can be written from (3.1), as follow:

$$
\ell \propto\left(\prod_{i=1}^{m} \frac{c \beta x_{i m: n}{ }^{(c-1)}}{\left(1+x_{i: m n n}^{c}\right)^{\beta+1}}\right)\left(\prod_{I=1}^{j} \frac{1}{\left(1+x_{i: m n n}^{c}\right)^{\beta}}\right)^{R_{i}}\left(\frac{1}{\left(1+x_{\mathrm{m} m: n}^{c}\right)^{\beta}}\right)^{n-m-\sum_{=1}^{j} R_{i}}
$$

Taking logarithm of $\ell$ gives: 


$$
\begin{aligned}
& L=\log \ell=m \log c+m \log \beta+(c-1) \\
& \sum_{i=1}^{m} \log x_{i: m: n}-(\beta+1) \sum_{i=1}^{m} \log \left(1+x_{i: m: n}^{c}\right) \\
& -\beta \sum_{i=1}^{j} R_{i} \log \left(1+x_{i: m: n}^{c}\right) \\
& -\beta\left(n-m-\sum_{i=1}^{j} R_{i}\right) \log \left(1+x_{m: m: n}^{c}\right)
\end{aligned}
$$

The point estimation for $c$ and $\beta$ can obtain by differentiating (3.3) to $c$ and $\beta$; respectively and equating the new equations to zero, yield:

$$
\begin{aligned}
& \frac{\partial L}{\partial c}=\frac{m}{c}+\sum_{i=1}^{m} \log x_{i: m: n}-(\beta+1) \sum_{i=1}^{m} \frac{x_{i: m: n}^{c} \log x_{i: m: n}}{1+x_{i: m: n}^{c}} \\
& -\beta \sum_{i=1}^{j} R_{i} \log \frac{x_{i: m: n}^{c} \log x_{i: m: n}}{1+x_{i: m: n}^{c}} \\
& -\beta\left(n-m-\sum_{i=1}^{j} R_{i}\right) \frac{x_{m: m: n}^{c} \log x_{m: m: n}}{1+x_{m: m: n}^{c}}=0 \\
& \frac{\partial L}{\partial \beta}=\frac{m}{\beta}-\sum_{i=1}^{m} \log \left(1+x_{i: m: n}^{c}\right)+\sum_{i=1}^{j} R_{i} \log \left(1+x_{i: m: n}^{c}\right) \\
& -\left(n-m-\sum_{i=1}^{j} R_{i}\right) \log \left(1+x_{m: m: n}^{c}\right)=0
\end{aligned}
$$

Solving Equation 3.4 and 3.5 to obtain $\hat{c}$ and $\hat{\beta}$, it's clear that, the analytical solution may be very difficult to find. So, the numerical methods are used to solve these equations. Also, the corresponding MLE of $S(t)$ and $H(t)$ can be written as:

$$
\begin{aligned}
& \hat{H}(x)=\frac{\hat{c} \hat{\beta} x^{(\hat{c}-1)}}{\left(1+x^{\hat{c}}\right)} \\
& \hat{S}(x)=\frac{1}{\left(1+x^{\hat{c}}\right)^{\hat{\beta}}}
\end{aligned}
$$

\section{Asymptotic Confidence Intervals}

In this section, the approximate confidence interval for $c, \beta, S(t)$ and $H(t)$ are obtained. $c, \beta$ was approximately bivariate normal with mean $(c, \beta)$ and variance-covariance matrix $I_{0}^{-1}$, Greene (2000; Agresti, 2002).

Where:

$$
I_{0}(c, \beta)=\left[\begin{array}{cc}
-\frac{\partial^{2} L}{\partial c^{2}} & \frac{\partial^{2} L}{\partial c \partial \beta} \\
\frac{\partial^{2} L}{\partial \beta \partial c} & \frac{\partial^{2} L}{\partial \beta^{2}}
\end{array}\right]
$$

The variance-covariance matrix is:

$$
I_{0}^{-1}(c, \beta)=\left[\begin{array}{cc}
\operatorname{var} \hat{c} & \operatorname{covar}(\hat{c}, \hat{\beta}) \\
\operatorname{covar}(\hat{c}, \hat{\beta}) & \operatorname{var} \hat{\beta}
\end{array}\right]
$$

The (1- $\alpha) \%$ approximate confidence interval for $c, \beta$, $S(t)$ and $H(t)$; respectively:

$$
\begin{aligned}
& \left(\hat{c}-Z_{\frac{\alpha}{2}} \sqrt{\operatorname{var} \hat{c}}, \hat{c}+Z_{\frac{\alpha}{2}} \sqrt{\operatorname{var} \hat{c}}\right), \\
& \left(\hat{\beta}-Z_{\frac{\alpha}{2}} \sqrt{\operatorname{var} \hat{\beta}}, \hat{\beta}+Z_{\frac{\alpha}{2}} \sqrt{\operatorname{var} \hat{\beta}}\right), \\
& \left(\hat{S}(t)-Z_{\frac{\alpha}{2}} \sqrt{\operatorname{var} \hat{S}(t)}, \hat{S}(t)+Z_{\frac{\alpha}{2}} \sqrt{\operatorname{var} \hat{S}(t)}\right), \\
& \left(\hat{H}(t)-Z_{\frac{\alpha}{2}} \sqrt{\operatorname{var} \hat{H}(t)}, \hat{H}(t)+Z_{\frac{\alpha}{2}} \sqrt{\operatorname{var} \hat{H}(t)}\right)
\end{aligned}
$$

where, $\operatorname{var}(c)$ and $\operatorname{var}(\beta)$ are the variances of the unknown parameters $c$ and $\beta$ which obtained from inverse fisher information matrix and $Z_{\frac{\alpha}{2}}$ is the percentile of the two tail probability $Z_{\frac{\alpha}{2}}$ calculated from standard normal distribution.

The $\sqrt{\operatorname{var} \hat{H}(t)}$ and $\sqrt{\operatorname{var} \hat{S}(t)}$ can be calculated from the next equations:

$\sqrt{\operatorname{varh}(t)} \simeq\left[G_{1}^{T} I_{0}^{-1} G_{1}\right]_{(\hat{c}, \hat{\beta})}$

And:

$\sqrt{\operatorname{var} \hat{S}(t)} \simeq\left[G_{2}^{T} I_{0}^{-1} G_{2}\right]_{(\hat{c}, \hat{\beta})}$

where, $\quad G_{1}=\left(\frac{\partial H(t)}{\partial c} \frac{\partial H(t)}{\partial \beta}\right), G_{2}=\left(\frac{\partial S(t)}{\partial c} \frac{\partial S(t)}{\partial \beta}\right) \quad$ and $I_{0}^{-1}$ is the variance-covariance matrix defined in (4.2):

\section{Bayes Estimation}

This section deals with estimating unknown parameters of Burr-XII distribution using Bayesian procedure. First, let the parameters $c$ and $\beta$ are independent and have gamma distributions as prior functions in the form; respectively:

$\pi_{1}(c)=\frac{b^{a} c^{a-1} e^{-b c}}{\Gamma(a)}, c>0$

And:

$\pi_{2}(\beta)=\frac{d^{k} \beta^{k-1} e^{-d \beta}}{\Gamma(k)}, \beta>0$ 
The joint prior distribution of $c$ and $\beta$ can be given as:

$$
g(c, \beta)=\ell(c, \beta \mid \underline{x}) \times \pi_{1}(c) \times \pi_{2}(\beta)
$$

The joint posterior density function can be written as:

$$
\pi(c, \beta \mid \underline{x})=\frac{\ell(c, \beta \mid \underline{x}) \times \pi_{1}(c) \times \pi_{2}(\beta)}{\int_{0}^{\infty} \int_{0}^{\infty} \ell(\underline{x} \mid c, \beta) \times \pi_{1}(c) \times \pi_{2}(\beta) d c d \beta}
$$

The Bayes estimator of any function under squared error loss function is the posterior mean and giving by:

$$
\begin{aligned}
& \hat{g}(c, \beta \mid \underline{x})=E(g(c, \beta \mid \underline{x})) \\
& =\frac{\int_{0}^{\infty} \int_{0}^{\infty} g(c, \beta) \ell((c, \beta \mid \underline{x})) \times \pi_{1}(c) \times \pi_{2}(\beta) d c d \beta}{\int_{0}^{\infty} \int_{0}^{\infty} \ell(c, \beta \mid \underline{x}) \times \pi_{1}(c) \times \pi_{2}(\beta) d c d \beta}
\end{aligned}
$$

In general, Equation 5.5 can't reduce to closed form. The exact solution may be very hard. In this case, the MCMC method to generate sample of (5.4) maybe the best procedure to compute Bayes estimator of $g(c, \beta)$.

Next section shows the MCMC approach including Metropolis-Hastings within Gibbs Sampling technic.

\section{Bayesian Estimation Using MCMC Approach}

The Metropolis-Hastings algorithm is a very general MCMC method developed by Metropolis et al. (1953) and extended by Hastings (1970). It's clear that, the solution of (5.5) is very hard to calculate, so, the MCMC maybe suitable method to estimate $\hat{c}$ and $\hat{\beta}$ by Bayesian approach. In order to use the method of MCMC for estimating the parameters of the Burr-XII distribution, let us consider independent priors (5.1 and 5.2), respectively, for the parameters $c$ and $\beta$. The joint posterior density function can be obtained up to proportionality by multiplying the likelihood with the prior and this can be written as:

$$
\begin{aligned}
& \pi(c, \beta \mid \underline{x}) \propto \beta^{m+k-1} c^{m+a-1} e^{-b c} e^{-d \beta}\left(\prod_{i=1}^{m} \frac{x_{i: m: n}^{(c-1)}}{\left(1+x_{i: m: n}^{c}\right)^{\beta+1}}\right) \\
& \times\left(\prod_{i=1}^{j} \frac{1}{\left(1+x_{i: m: n}^{c}\right)^{\beta}}\right)^{R_{i}}\left(\frac{1}{\left(1+x_{m: m: n}^{c}\right)^{\beta}}\right)^{n-m-\sum_{i=1}^{j} R_{i}}
\end{aligned}
$$

From (6.1), the posterior density function of $c$ given $\beta$ and $\beta$ given $c$ are, respectively:

$$
\begin{aligned}
& g_{1}(c \mid \beta, \underline{x}) \propto c^{m+a-1} e^{-b c}\left(\prod_{i=1}^{m} \frac{x_{i: m: n}^{(c-1)}}{\left(1+x_{i: m: n}^{c}\right)^{\beta+1}}\right) \\
& \times\left(\prod_{i=1}^{j} \frac{1}{\left(1+x_{i: m: n}^{c}\right)^{\beta}}\right)^{R_{i}} \times\left(\frac{1}{\left(1+x_{m: m: n}^{c}\right)^{\beta}}\right)^{n-m-\sum_{i=1}^{j} R_{i}}
\end{aligned}
$$

And:

$$
\begin{aligned}
& g_{2}(\beta \mid c, \underline{x}) \propto \beta^{m+k-1} e^{-d \beta}\left(\prod_{i=1}^{m} \frac{x_{i: m: n}^{(c-1)}}{\left(1+x_{i: m: n}^{c}\right)^{\beta+1}}\right) \\
& \times\left(\prod_{i=1}^{j} \frac{1}{\left(1+x_{i: m: n}^{c}\right)^{\beta}}\right)^{R_{i}}\left(\frac{1}{\left(1+x_{m: m: n}^{c}\right)^{\beta}}\right)^{n-m-\sum_{i=1}^{j} R_{i}}
\end{aligned}
$$

It's clear that the posterior density function of $c$ and $\beta$ given in (6.2 and 6.3) cannot be reduced analytically to well known distributions. The Metropolis-Hastings method with normal proposal distribution will be used to generate $c$ and $\beta$ from the posterior density functions and in turn obtain the Bayes estimates and the corresponding credible intervals can be written as:

- Start with $\beta^{(0)}$

- Set $v=1$

- Using Metropolis-Hastings (Metropolis et al., 1953), generate $c^{v}$ from $g_{1}$ with the normal $N\left(c^{v}, \sigma^{2}\right)$. Where $\sigma^{2}$ is variances-covariance matrix

- Also, generate $\beta^{v}$ from $g_{2}$ with the normal $N\left(\beta^{\nu-1}, \sigma^{2}\right)$

- Compute $c^{v}$ and $\beta^{v}$

- $\quad$ Set $v=v+1$

- Repeat 3-6 steps $\mathrm{N}$ times

- Rearrange the values $c^{i}$ and $\beta^{i}, i=M+1, M+2, \cdots, N$

- Obtain the Bayes estimates of $\mathrm{c}$ and $\beta$ with respect to the squared error loss function as:

$$
E(c \mid \text { data })=\frac{1}{N-M} \sum_{i=M+1}^{N} c_{i}
$$

and

$$
E(\beta \mid \text { data })=\frac{1}{N-M} \sum_{i=M+1}^{N} \beta_{i} \text {, where } \mathrm{M} \text { is burn-in }
$$

- $(1-\alpha) \%$ credible intervals of $c$ and $\beta$ can be computed as:

$$
\left(c_{\left\{\frac{\alpha}{2}(N-M)\right\}}, c_{\left\{\frac{1-\alpha}{2}(N-M)\right\}}\right) \text { and }\left[\beta_{\left\{\frac{\alpha}{2}(N-M)\right\}}, \beta_{\left\{\frac{1-\alpha}{2}(N-M)\right\}}\right]
$$

\section{Numerical Results}

This section deals with obtaining some numerical results. Confidence interval for $\mathrm{c}$ and $\beta$ using maximum likelihood and Bayes include MCMC technique are calculated. In order to compare the different estimators 
of the parameters, 11000 an adaptive Type-II progressive samples from the Burr-XII distribution with different censoring schemes $\mathrm{R}$ are simulated. The samples were simulated by using the algorithm described in $\mathrm{Ng}$ et al. (2010). Compare the performances of ML and Bayesian estimates in terms of Mean Squared Errors (MSEs). To find the Bayesian MCMC estimates, the non-informative gamma priors is used, call it prior 0 . In addition, an informative gamma priors for the two parameters call it priorl is used.

Table 1. Censoring schemes with different values for $n$ and $m$

\begin{tabular}{|c|c|c|c|}
\hline Censoring scheme & $n$ & $m$ & $R$ \\
\hline $\mathrm{CS}_{1}$ & 50 & 30 & $\mathrm{R}_{1}=\left\{0^{8}, 2,0^{6}, 8,3,0^{3}, 5,0^{8}, 2\right\}$ \\
\hline $\mathrm{CS}_{2}$ & 50 & 30 & $\mathrm{R}_{2}=\left\{0^{4}, 1,0^{6}, 3,2,0^{2}, 8,3,0^{3}, 3,0^{9}\right\}$ \\
\hline $\mathrm{CS}_{3}$ & 60 & 30 & $\mathrm{R}_{3}=\left\{0^{3}, 5,0,8,0^{2}, 2,0^{2}, 7,0^{3}, 1,3,0^{3}, 1,0^{5}, 1,0^{2}, 2\right\}$ \\
\hline $\mathrm{CS}_{4}$ & 60 & 30 & $\mathrm{R}_{4}=\left\{0^{2}, 5,0,4,0^{4}, 3,0^{3}, 2,0,3,0^{3}, 5,0^{3}, 3,0^{2}, 5,0^{3}\right\}$ \\
\hline $\mathrm{CS}_{5}$ & 60 & 40 & $\mathrm{R}_{5}=\left\{0^{8}, 2,0^{3}, 6,0^{2}, 0^{5}, 3,0^{2}, 2,0^{4}, 1^{2}, 0^{3}, 2,0^{3}, 2\right\}$ \\
\hline $\mathrm{CS}_{6}$ & 60 & 40 & $\mathrm{R}_{6}=\left\{0^{4}, 3,0^{5}, 2,0^{4}, 2,3,0^{3}, 5,0^{8}, 2,1,0^{8}, 2\right\}$ \\
\hline $\mathrm{CS}_{7}$ & 60 & 50 & $\mathrm{R}_{7}=\left\{0^{8}, 2,0^{6}, 1,3,0^{3}, 1,0^{8}, 2,1,0^{19}\right\}$ \\
\hline $\mathrm{CS}_{8}$ & 60 & 50 & $\mathrm{R}_{8}=\left\{0^{8}, 2,0^{6}, 3,0^{19}, 0^{10}, 2,0^{3}\right\}$ \\
\hline $\mathrm{CS}_{9}$ & 70 & 50 & $\mathrm{R}_{9}=\left\{0^{8}, 2,0^{3}, 3,0^{3}, 3,0^{3}, 7,0^{8}, 2,3,0^{19}\right\}$ \\
\hline $\mathrm{CS}_{10}$ & 70 & 50 & $\mathrm{R}_{10}=\left\{0^{8}, 2,0^{6}, 8,3,0^{3}, 5,0^{8}, 2,0^{20}\right\}$ \\
\hline $\mathrm{CS}_{11}$ & 70 & 60 & $\mathrm{R}_{11}=\left\{0^{8}, 2,0^{6}, 1,3,0^{12}, 2,0^{8}, 2,0^{21}\right\}$ \\
\hline $\mathrm{CS}_{12}$ & 70 & 60 & $\mathrm{R}_{12}=\left\{0^{15}, 2,0^{4}, 5,0^{18}, 3,0^{20}\right\}$ \\
\hline
\end{tabular}

Remark: $0^{r}$ means that 0 repeated $r$ times.

Table 2. Mean, Interval Length (IL) for $c$ and $\beta$ using Maximum Likelihood method (ML)

\begin{tabular}{|c|c|c|c|c|c|c|c|c|c|c|c|c|c|}
\hline & & Censo & scheme & & & & & & & & & & \\
\hline & & $\mathrm{CS}_{1}$ & $\mathrm{CS}_{2}$ & $\mathrm{CS}_{3}$ & $\mathrm{CS}_{4}$ & $\mathrm{CS}_{5}$ & $\mathrm{CS}_{6}$ & $\mathrm{CS}_{7}$ & $\mathrm{CS}_{8}$ & $\mathrm{CS}_{9}$ & $\mathrm{CS}_{10}$ & $\mathrm{CS}_{11}$ & $\mathrm{CS}_{12}$ \\
\hline$\hat{\hat{c}}$ & mean & 3.1068 & 3.2235 & 3.131 & 3.2061 & 3.0988 & 3.1953 & 3.0756 & 3.0772 & 3.1024 & 3.0443 & 3.0992 & 3.1028 \\
\hline & MSE & 0.2723 & & & & & & & & & & & \\
\hline & IL & 2.1949 & & & & & & & & & & & \\
\hline & cov & 0.9800 & 0.9700 & 0.9200 & 0.9800 & 0.9700 & 0.9900 & 0.9700 & 0.9500 & 0.9600 & 0.9500 & 0.9800 & 0.9700 \\
\hline$\hat{\beta}$ & mean & 0.4995 & 0.5167 & 0.5330 & 0.5097 & 0.5111 & 0.4957 & 0.5057 & 0.5122 & 0.4945 & 0.5136 & 0.4903 & 0.4949 \\
\hline & MSE & & & & & & & & & & & & \\
\hline & IL & 0.4092 & 0.4191 & 0.4097 & 0.3968 & & 0.3666 & & 0.3620 & & & & 0.3267 \\
\hline & $\mathrm{cov}$ & 0.9600 & 0.9100 & 0.9600 & 0.9600 & 0.9800 & & & & 0.9100 & & & 0.9200 \\
\hline $\mathrm{S}^{\wedge}(\mathrm{t})$ & mean & 0.9684 & 0.9693 & 0.9664 & 0.9705 & 0.9680 & 0.9714 & 0.9677 & 0.9672 & 0.9688 & 0.9667 & 0.9696 & 0.9688 \\
\hline & MSE & & & & & & & & & & & & 0.0158 \\
\hline & IL & 0 . & 0.06 & 0.06 & & & 0.0592 & & & & & & 0.0578 \\
\hline & cov & & 0.8800 & 0.8800 & & & 0.8800 & & & & & & 0.8800 \\
\hline $\mathrm{H}^{\wedge}(\mathrm{t})$ & mean & 0.2226 & 0.2194 & 0.2359 & 0.2147 & 0.2266 & 0.2100 & 0.2282 & 0.2314 & 0.2204 & 0.2336 & 0.2180 & 0.2212 \\
\hline & MSE & 0.0881 & 0.0972 & 0.0933 & 0.0834 & 0.0831 & 0.0822 & 0.0794 & 0.0872 & 0.0810 & 0.0800 & 0.0780 & 0.0826 \\
\hline & IL & & & & & & & & & & & & 0.0327 \\
\hline & cov & 0.9200 & 0.9000 & 0.9000 & 0.9100 & 0.9200 & 0.9200 & 0.9300 & 0.8900 & 0.9000 & 0.9600 & 0.9000 & 0.8900 \\
\hline
\end{tabular}

Table 3. Mean, Interval Length (IL) for $c$ and $\beta$ using Bayesian method (mcmc1)

\begin{tabular}{|c|c|c|c|c|c|c|c|c|c|c|c|c|c|}
\hline & & \multicolumn{12}{|c|}{ Censoring scheme } \\
\hline & & $\mathrm{CS}_{1}$ & $\mathrm{CS}_{2}$ & $\mathrm{CS}_{3}$ & $\mathrm{CS}_{4}$ & $\mathrm{CS}_{5}$ & $\mathrm{CS}_{6}$ & $\mathrm{CS}_{7}$ & $\mathrm{CS}_{8}$ & $\mathrm{CS}_{9}$ & $\mathrm{CS}_{10}$ & $\mathrm{CS}_{11}$ & $\mathrm{CS}_{12}$ \\
\hline \multirow[t]{4}{*}{$\hat{c}$} & mean & 2.9655 & 3.0755 & 3.0039 & 3.052 & 2.9993 & 3.0529 & 2.9543 & 2.9402 & 2.9988 & 2.9251 & 2.9946 & 2.9800 \\
\hline & MSE & 0.2787 & 0.5322 & 0.3832 & 0.3814 & 0.3465 & 0.3294 & 0.2921 & 0.2281 & 0.2662 & 0.2274 & 0.1954 & 0.3220 \\
\hline & IL & 1.1237 & 1.0819 & 1.0364 & 1.0518 & 0.9601 & 1.0037 & 0.9694 & 0.9610 & 0.9318 & 0.9037 & 0.8870 & 0.8507 \\
\hline & $\operatorname{cov}$ & 0.6500 & 0.5400 & 0.5200 & 0.6200 & 0.5500 & 0.5700 & 0.6200 & 0.6400 & 0.6300 & 0.6400 & 0.6700 & 0.5800 \\
\hline \multirow[t]{4}{*}{$\hat{\beta}$} & mean & 0.5189 & 0.5371 & 0.5465 & 0.5261 & 0.5274 & 0.5163 & 0.5263 & 0.5342 & 0.5103 & 0.5321 & 0.5077 & 0.5161 \\
\hline & MSE & 0.0117 & 0.0177 & 0.0128 & 0.0089 & 0.0100 & 0.0104 & 0.0100 & 0.0110 & 0.0087 & 0.0099 & 0.0082 & 0.0094 \\
\hline & IL & 0.3842 & 0.3958 & 0.3932 & 0.3810 & 0.3401 & 0.3360 & 0.3203 & 0.3251 & 0.3018 & 0.3141 & 0.2856 & 0.2855 \\
\hline & $\operatorname{cov}$ & 0.9200 & 0.8600 & 0.9600 & 0.9900 & 0.9400 & 0.9000 & 0.8900 & 0.8700 & 0.8400 & 0.8800 & 0.8600 & 0.8600 \\
\hline \multirow[t]{4}{*}{$\mathrm{S}^{\wedge}(\mathrm{t})$} & mean & 0.9628 & 0.9617 & 0.9607 & 0.9644 & 0.9624 & 0.9650 & 0.9619 & 0.9614 & 0.9642 & 0.9613 & 0.9653 & 0.9626 \\
\hline & MSE & 0.0201 & 0.0260 & 0.0240 & 0.0200 & 0.0222 & 0.0199 & 0.0205 & 0.0212 & 0.021 & 0.0201 & 0.0178 & 0.0217 \\
\hline & IL & 0.0774 & 0.0766 & 0.0729 & 0.0695 & 0.0699 & 0.0673 & 0.0710 & 0.0715 & 0.0632 & 0.0667 & 0.0625 & 0.0646 \\
\hline & $\operatorname{cov}$ & 0.9300 & 0.8500 & 0.9300 & 0.9100 & 0.9300 & 0.9100 & 0.9200 & 0.9300 & 0.9000 & 0.9100 & 0.9100 & 0.8800 \\
\hline \multirow[t]{4}{*}{$\mathrm{H}^{\wedge}(\mathrm{t})$} & mean & 0.2489 & 0.2512 & 0.2589 & 0.2413 & 0.2494 & 0.2388 & 0.2540 & 0.2585 & 0.2408 & 0.2584 & 0.2386 & 0.2481 \\
\hline & MSE & 0.0972 & 0.1197 & 0.1102 & 0.0937 & 0.1014 & 0.0977 & 0.0974 & 0.1004 & 0.0943 & 0.0943 & 0.0858 & 0.1008 \\
\hline & IL & 0.3748 & 0.3703 & 0.3495 & 0.3426 & 0.3397 & 0.3363 & 0.3443 & 0.3491 & 0.3122 & 0.3226 & 0.3143 & 0.3137 \\
\hline & $\operatorname{cov}$ & 0.9300 & 0.8600 & 0.9400 & 0.9000 & 0.9000 & 0.9100 & 0.9200 & 0.9000 & 0.8700 & 0.9200 & 0.9300 & 0.8800 \\
\hline
\end{tabular}




\begin{tabular}{|c|c|c|c|c|c|c|c|c|c|c|c|c|c|}
\hline & & \multicolumn{12}{|c|}{ Censoring scheme } \\
\hline & & $\mathrm{CS}_{1}$ & $\mathrm{CS}_{2}$ & $\mathrm{CS}_{3}$ & $\mathrm{CS}_{4}$ & $\mathrm{CS}_{5}$ & $\mathrm{CS}_{6}$ & $\mathrm{CS}_{7}$ & $\mathrm{CS}_{8}$ & $\mathrm{CS}_{9}$ & $\mathrm{CS}_{10}$ & $\mathrm{CS}_{11}$ & $\mathrm{CS}_{12}$ \\
\hline \multirow[t]{4}{*}{$\hat{c}$} & mean & 3.0734 & 3.1983 & 3.1837 & 3.1383 & 3.0805 & 3.1747 & 3.0991 & 3.0468 & 3.0875 & 3.0194 & 3.0635 & 3.0571 \\
\hline & MSE & 0.3650 & 0.4544 & 0.5333 & 0.3339 & 0.3265 & 0.3528 & 0.3594 & 0.3145 & 0.3628 & 0.2654 & 0.2366 & 0.3440 \\
\hline & IL & 1.1341 & 1.1668 & 1.0806 & 1.1073 & 1.0262 & 1.0003 & 0.9970 & 0.9777 & 0.9260 & 0.9119 & 0.8714 & 0.8596 \\
\hline & $\operatorname{cov}$ & 0.6300 & 0.6400 & 0.6000 & 0.6100 & 0.5700 & 0.6200 & 0.6100 & 0.5600 & 0.4800 & 0.6600 & 0.6000 & 0.5600 \\
\hline \multirow[t]{4}{*}{$\hat{\beta}$} & mean & 0.5045 & 0.5204 & 0.5289 & 0.5142 & 0.5149 & 0.5011 & 0.5082 & 0.521 & 0.4993 & 0.5191 & 0.4976 & 0.5047 \\
\hline & MSE & 0.0110 & 0.0163 & 0.0121 & 0.0087 & 0.0089 & 0.0110 & 0.0105 & 0.0123 & 0.0085 & 0.0085 & 0.0082 & 0.0093 \\
\hline & IL & 0.3794 & 0.3896 & 0.3888 & 0.3771 & 0.3412 & 0.3309 & 0.3135 & 0.3195 & 0.2971 & 0.3114 & 0.2781 & 0.2817 \\
\hline & $\operatorname{cov}$ & 0.9100 & 0.8800 & 0.9500 & 0.9700 & 0.9200 & 0.8700 & 0.8700 & 0.8200 & 0.8600 & 0.9100 & 0.8400 & 0.8700 \\
\hline \multirow[t]{4}{*}{$\mathrm{S}^{\wedge}(\mathrm{t})$} & mean & 0.9659 & 0.9678 & 0.9664 & 0.9681 & 0.9661 & 0.9690 & 0.9664 & 0.9639 & 0.9666 & 0.9649 & 0.9674 & 0.9655 \\
\hline & MSE & 0.0207 & 0.0200 & 0.0200 & 0.0178 & 0.0192 & 0.0188 & 0.0191 & 0.0222 & 0.0192 & 0.0178 & 0.0169 & 0.0193 \\
\hline & IL & 0.0718 & 0.0689 & 0.0655 & 0.0645 & 0.0653 & 0.0616 & 0.0647 & 0.0672 & 0.0599 & 0.0624 & 0.0596 & 0.0611 \\
\hline & $\operatorname{cov}$ & 0.9000 & 0.8400 & 0.8100 & 0.8700 & 0.8900 & 0.8700 & 0.8600 & 0.8500 & 0.8500 & 0.9300 & 0.8700 & 0.8800 \\
\hline \multirow[t]{4}{*}{$\mathrm{H}^{\wedge}(\mathrm{t})$} & mean & 0.2308 & 0.2263 & 0.2329 & 0.2244 & 0.2331 & 0.2188 & 0.2314 & 0.2436 & 0.2281 & 0.2411 & 0.2274 & 0.2346 \\
\hline & MSE & 0.0984 & 0.1028 & 0.1029 & 0.0872 & 0.0927 & 0.0965 & 0.0965 & 0.1068 & 0.0934 & 0.0865 & 0.0861 & 0.0955 \\
\hline & IL & 0.0326 & 0.0308 & 0.0370 & 0.0306 & 0.0357 & 0.0312 & 0.0359 & 0.0423 & 0.0376 & 0.0405 & 0.0355 & 0.0403 \\
\hline & $\operatorname{cov}$ & 0.9000 & 0.8800 & 0.8700 & 0.9100 & 0.9000 & 0.8600 & 0.8700 & 0.8500 & 0.8900 & 0.9500 & 0.8800 & 0.9000 \\
\hline
\end{tabular}

Table 5. Maximum likelihood and non-informative Bayesian including Markov chain Mont Carlo simulation (mcmc0) For real data set

\begin{tabular}{llll}
\hline ML & Mean & Lower & Upper \\
\hline$c$ & 1.2739 & 0.3836 & 2.1642 \\
$\beta$ & 0.2484 & 0.0370 & 0.4599 \\
$S$ & 0.7369 & 0.5883 & 0.8854 \\
$H$ & 0.1120 & 0.0350 & 0.1889 \\
mcmc0 & & & \\
$c$ & 1.3163 & 0.5882 & 2.3965 \\
$\beta$ & 0.2581 & 0.0938 & 0.5282 \\
$S$ & 0.7242 & 0.5647 & 0.8837 \\
$H$ & 0.1212 & 0.0392 & 0.2032 \\
\hline
\end{tabular}

The Bayesian estimates and probability intervals based on 11000 MCMC samples and discard the first 1000 values as burn-in is computed. All results are given in Tables are obtained at $c=3 ; \beta=0.5 ; T=0.5$; $t=0.4 ; \alpha=0.05 ; a=0.5 ; b=1 ; \gamma=0.8 ; \xi=1 ;$ nmcmc $=11000 ;$ nburn $=1000 ; a 1=0 ; b 1=0 ; \xi_{1}=0 ; \gamma_{1}=0$ and different censoring schemes (Table 1).

where, $0^{r}$ means that 0 repeated $r$ times.

\section{Remarks}

From Table 2-4, observe that:

- When $n$ and $m$ increase, the MSE specially in mcmc 1 and ML decrease

- In mcmc1 and mcmc0, the coverage probability is small than ML for parameter $c$

- Based on the interval length and the mean squared error. The momcl is the best method for estimating all parameters except the parameter $c$

\section{Illustrative Example}

The data set was discussed here are presented by (Zimmer et al., 1998; Lio et al., 2010) for the Burr-XII reliability analysis. Lio et al. (2010) studied the validity of the model for both data sets and they showed that Burr-XII distribution fits quite well for both the data sets. These data sets are $0.19,0.78,0.96,0.31,2.78$, $3.16,4.15,4.67,4.85,6.50,7.35,8.01,8.27,12.06$, $31.75,32.52,33.91,36.71$ and 72.89 . It is clear that $n=$ 19. Suppose that, $m=10 ; \alpha=0.05$ (significant level); $t=$ $2 ;$ nmcmc $=11000 ;$ nburn $=1000, R=1,0,0,2,0,3,0,0$, 1,2 . If $T=7.5$ then $J=8$ and $R=1,0,0,2,0,3,0,0,0,3$ then $x_{i}(i=1,2, \cdots, m)=0.19,0.31,0.96,2.78,3.16,4.15$, $4.67,6.5,8.27,31.75$. Because no prior information about unknown parameters. The results are calculated here using mathematica 8 and posted in Table 5 using maximum likelihood and non-informative Bayesian including Markov chain Mont Carlo simulation ( $\mathrm{mcmc} 0)$.

\section{Conclusion}

In this study, the Maximum Likelihood Estimation (MLE) and Bayes estimation are exploited to make interval estimation for the unknown parameters of BurrXII distribution. Bayes estimator does not exist in an explicit form for the parameters, Markov Chain Monte Carlo (MCMC) method is used to generate samples from the posterior distribution. Numerical example using real data set is presented to illustrate the methods of inference developed here.

\section{Acknowledgement}

The author is grateful to the referees for their comments and suggestions, which certainly improved the presentation of the content of the paper.

\section{Ethics}

This article is original and contains unpublished material. The corresponding author confirms that all of the other authors have read and approved the manuscript and no ethical issues involved. 


\section{References}

Aggarwala, R. and N. Balakrishnan, 1996. Recurrence relations for single and product moments of progressive Type-II right censored order statistics from exponential and truncated exponential distributions. Ann. Inst. Stat. Math., 48: 757-771. DOI: $10.1007 / \mathrm{BF} 00052331$

Agresti, A., 2002. Categorical Data Analysis. 2nd Edn., Wiley, New York, ISBN-10: 0471360937, pp: 734.

Ali Mousa, M.A.M. and Z.F. Jaheen, 2002. Statistical inference for the Burr model based on progressively censored data. Comput. Math. Applic., 43: 1441-1449. DOI: 10.1016/S0898-1221(02)00110-4

Balakrishnan, N. and R. Aggarwala, 2000. Progressive Censoring: Theory, Methods and Applications. 1st Edn., Springer Science and Business Media, Boston, ISBN-10: 0817640010, pp: 248.

Balakrishnan, N., 2007. Progressive censoring methodology: An appraisal. Test, 16: 211-296. DOI: $10.1007 / \mathrm{s} 11749-007-0061-y$

Balakrishnan, N., A. Childs and B. Chandrasekar, 2002. An efficient computational method for moments of order statistics under progressive censoring. Stat. Probab. Lett., 60: 359-365. DOI: $10.1016 / \mathrm{S} 0167-7152(02) 00267-5$

Burr, I.W., 1942. Cumulative frequency functions. Ann. Math. Stat., 13: 215-232.

Greene, W.H., 2000. Econometric Analysis. 4th Edn., Prentice Hall, New York, ISBN-10: 0130132977, pp: 1004.

Hastings, W.K., 1970. Monte Carlo sampling methods using Markov chains and their applications. Biometrika, 57: 97-109. DOI: 10.2307/2334940

Jaheen, Z.F. and H.M. Okasha, 2011. E-Bayesian estimation for the Burr type XII model based on type-2 censoring. Applied Math. Modell., 35: 4730-4737. DOI: 10.1016/j.apm.2011.03.055

Lio, Y.L., T.R. Tsai and S.J. Wu, 2010. Acceptance sampling plans from truncated life tests based on the Burr type XII percentiles. J. Chinese Inst. Indust. Eng., 27: 270-280.

DOI: $10.1080 / 10170661003791029$

Mahmoud, M.A.W., A.A. Soliman, A.H.A. Ellah and R.M. El-Sagheer, 2013. Estimation of generalized pareto under an adaptive Type-II progressive censoring. Intell. Inform. Manage., 5: 73-83. DOI: $10.4236 /$ iim.2013.53008

Metropolis, N., A.W. Rosenbluth, M.N. Rosenbluth, A.H. Teller and E. Teller, 1953. Equation of state calculations by fast computing machines. J. Chem. Phys., 21: 1087-1091. DOI: 10.1063/1.1699114

Moore, D. and A.S. Papadopoulos, 2000. The Burr type XII distribution as a failure model under various loss functions. Microelectron. Reliab., 40: 2117-2122. DOI: 10.1016/S0026-2714(00)00031-7
Ng, H.K.T. and P.S. Chan, 2007. Discussion on progressive censoring methodology: An appraisal by N. Balakrishnan. Test, 16: 287-289. DOI: $10.1007 / \mathrm{s} 11749-007-0061-y$

Ng, H.K.T. Kundu, D. and P.S. Chan, 2010. Statistical analysis of exponential lifetimes under an adaptive Type-II progressive censoring scheme. Naval Res. Logist., 56: 687-698. DOI: 10.1002/nav.20371

Rezaei, S., R. Tahmasbi and M. Mahmoodi, 2010. Estimation of $\mathrm{P}[\mathrm{Y}<\mathrm{X}]$ for generalized Pareto distribution. J. Stat. Plann. Inference, 140: 480-494. DOI: $10.1016 /$ j.jspi.2009.07.024

Robert, C.P. and G. Casella, 2005. Monte Carlo Statistical Methods. 2nd Edn., Springer, New York, ISBN-10: 0387212396, pp: 649.

Rodriguez, R.N., 1977. A guide to the burr type XII distributions. Biometrika, 64: 129-134. DOI: $10.2307 / 2335782$

Soliman, A.A., 2005. Estimation of parameters of life from progressively censored data using Burr-XII model. IEEE Trans. Reliab., 54: 34-42. DOI: 10.1109/TR.2004.842528

Soliman, A.A., A.H. Abd-Ellah, N.A. Abou-Elheggag and E.A. Ahmed, 2011. Reliability estimation in stress-strength models: An MCMC approach. Statistics, 47: 1-14. DOI: 10.1080/02331888.2011.637629

Soliman, A.A., A.H. Abd-Ellah, N.A. Abou-Elheggag and E.A. Ahmed, 2012. Modified Weibull model: A bayes study using MCMC approach based on progressive censoring data. Reliab. Eng. Syst. Safety, 100: 48-57. DOI: 10.1016/j.ress.2011.12.013

Viveros, R. and N. Balakrishnan, 1994. Interval estimation of parameters of life from progressively censored data. Technometrics, 36: 84-91. DOI: 10.1080/00401706.1994.10485403

Wingo, D.R., 1993. Maximum likelihood methods for fitting the burr type XII distribution to multiply (progressively) censored life test data. Metrika, 40: 203-210. DOI: 10.1007/BF02613681

Wu, J.W. and H.Y. Yu, 2005. Statistical inference about the shape parameter of the Burr type XII distribution under the failure-censored sampling plan. Applied Math. Computat., 163: 443-482.

DOI: 10.1016/j.amc.2004.02.019

Wu, J.W., W.C. Lee, C.W. Hong and S.Y. Yeh, 2014. Implementing lifetime performance index of burr xii products with progressively type ii right censored sample. Int. J. Innovative Comput. Inform. Control, 10: 671-693.

$\mathrm{Wu}$, S.J., 2002. Estimations of the parameters of the Weibull distribution with progressively censored data. J. Japan Stat. Society, 32: 155-163.

DOI: $10.14490 /$ jjss.32.155 
Xiuchun, L., S. Yimin, W. Jieqiong and C. Jian, 2007. Empirical Bayes estimators of reliability performances using LINEX loss under progressively Type-II censored samples. Math. Comput. Simulat., 73: 320-326. DOI: 10.1016/j.matcom.2006.05.002
Zimmer, W.J., J.B. Keats and F.K. Wang, 1998. The Burr XII distribution in reliability analysis. J. Q. Technol., 30: 386-394. 